\title{
System and Experiment on Fast Testing Vermicular Graphite Percent in Cast Iron Based on Ultrasonic Longitudinal Wave
}

\author{
Dequan Shi*, Kaijiao Kang, Guili Gao and Shen Miao \\ Department of Materials Science \& Engineering, Harbin University of Science and Technology, Harbin 150040, China
}

\begin{abstract}
Vermicular graphite percent is an important index of evaluating the vermiculation of graphite in cast iron. Quantitative metallographic method is commonly used to test the vermicular graphite percent, but it can only detect certain parts of the cast iron specimen. Therefore, an ultrasonic system was designed to detect the degree of the vermiculation in cast iron according to the relationship between the ultrasonic propagation velocity and the vermicular graphite percent, and it is composed of the sink filled with ultrasonic coupling agent, ultrasonic emitting probe, ultrasonic receiving probe, high-frequency signal generator, ultrasonic signal acquisition unit and computer. Without measuring the length of the cast iron specimen, the propagation velocity can be calculated by three different kinds of propagation time. A specific relation between the vermicular graphite percent $\eta$ and the propagation velocity $v$ was got by experiments. When the propagation velocity is below $5700 \mathrm{~m} \cdot \mathrm{s}^{-1}$, they have an approximate exponential relationship, namely $\eta=0.6 \cdot \exp (26800 / v)$. When the propagation velocity exceeds $5700 \mathrm{~m} \cdot \mathrm{s}^{-1}$, they have a linear relationship, namely $\eta=-v / 1.53+5800 / 1.53$. The comparative experiments showed that the coefficient of variation and the error were below $0.3 \%$ and $4 \%$, respectively, which indicated the repeatability and accuracy of the system are very good. [doi:10.2320/matertrans.M2015439]
\end{abstract}

(Received December 1, 2015; Accepted January 25, 2016; Published March 4, 2016)

Keywords: vermicular graphite cast iron, vermicular graphite percent, ultrasonic testing, quantitative metallographic

\section{Introduction}

The tensile strength of vermicular graphite cast iron is between the grey cast iron and nodular graphite cast iron, and its fluidity, feeding capacity and thermal fatigue resistance is better than that of nodular graphite cast iron. ${ }^{1,2)}$ In recent years, it has been widely used in the parts of automotive industry.

Vermicular graphite percent is not only the key index of determining the vermiculation of graphite in cast iron, but also is the important indicator of evaluating the quality and the technology level of vermicular graphite cast iron. ${ }^{3)}$ During the production process, in order to avoid a mass of flake graphite or spheroidal graphite arising from the inappropriate modification, there is a need to rapidly and accurately detect the vermicular graphite percent, and thus to adjust the quantity of the vermicular agent on time.

Quantitative metallographic method is the most commonly used to detect the vermicular graphite percent, but its detection speed is very slow, and what is the important is that it can only detect certain part of the cast iron specimen, which will not reflect the whole degree of the vermiculation of graphite in the specimen. ${ }^{4,5)}$ The previous researches had showed that there is a certain relationship between the ultrasonic velocity and the vermicular graphite percent in cast iron. ${ }^{6)}$ So, if a specific relationship between them was got, the vermicular graphite percent could be figured out by the ultrasonic velocity. However, most of the ultrasonic probes must be directly contacted with the detected specimen, and only when the distance of the ultrasonic propagation directing to the specimen was accurately measured, the ultrasonic velocity could be calculated. ${ }^{7-9)}$ In fact, the length of the detected specimen is often different, which will bring some difficulties to detect the vermicular graphite percent.

In order to solve the above problem, a system was designed

*Corresponding author, E-mail: shidequan2008@163.com to detect the vermicular graphite percent according to the ultrasonic longitudinal wave, and a specific relationship between the ultrasonic velocity and the vermicular graphite percent in cast iron was achieved by experiments. This system mainly includes the sink filled with ultrasonic coupling agent, ultrasonic emitting probe, ultrasonic receiving probe, high frequency signal generator, ultrasonic signal acquisition unit, and computer, and the software for the system was written by LabVIEW.

\section{Principle of Detecting Vermicular Graphite Percent Based on Ultrasonic Wave}

The ultrasonic wave can spread in the solid media such as the vermicular graphite cast iron. If the solid medium is infinite, the propagation velocity of the ultrasonic longitudinal wave can be calculated according to the eq. (1). ${ }^{10)}$

$$
V_{1}=\sqrt{\frac{E}{\rho} \times \frac{1-\mu}{1+\mu}(1-2 \mu)}
$$

where, $V_{1}$ is the propagation velocity of the longitudinal wave, $\mathrm{m} \cdot \mathrm{s}^{-1}, E$ is Young's elastic modulus, $\mathrm{N} / \mathrm{m}^{2}, \rho$ is the density of the detected specimen, $\mathrm{kg} / \mathrm{m}^{3}$, and $\mu$ the Poisson's ratio.

Because the density $\rho$ and Poisson's ratio $\mu$ of vermicular graphite cast iron have a small change, they have little influence on the propagation velocity $V_{1}$ of the longitudinal wave. So, according to the eq. (1), the propagation velocity $V_{1}$ is mainly determined by the Young's elastic modulus $E$ which has direct relation to the graphite shape. ${ }^{11,12)}$ As a result, the propagation velocity $V_{1}$ will be decided by the vermicular graphite percent if the carbon equivalent of cast iron is kept in a certain range. Once the vermiculation degree of graphite was too much low or high, the Young's elastic modulus and thus the propagation velocity would be rapidly changed with the number of flake graphite or spheroidal graphite. So, through measuring the propagation velocity of ultrasonic lon- 
gitudinal wave, the vermicular graphite percent in cast iron can be identified.

As shown in Fig. 1, the emitting and receiving probes are installed at two sides of the sink, respectively.

Before the vermicular graphite cast iron specimen was put into the sink filled with the coupling agent, the propagation time of ultrasonic wave in the coupling agent can be written as the following equation.

$$
t_{0}=\frac{L_{0}}{v_{0}}
$$

where, $t_{0}$ is the propagation time in the coupling agent, $L_{0}$ is the distance between the emitting probe and the receiving probe, and $v_{0}$ is the ultrasonic propagation velocity in the coupling agent.

After the specimen was put, the ultrasonic longitudinal wave from the emitting probe will propagate through the coupling agent and the vermicular graphite cast iron sample, and then will be received by the receiving probe, which was called as the first wave. In addition, the ultrasonic longitudinal wave will be also reflected twice by the specimen, and then arrive at the receiving probe, which was called as the second wave. The propagation time of the first wave and the second wave can be described as the following equations.

$$
\begin{aligned}
& t_{1}=\frac{L_{0}-L}{v_{0}}+\frac{L}{v} \\
& t_{2}=\frac{L_{0}-L}{v_{0}}+\frac{3 L}{v}
\end{aligned}
$$

where, $L$ is the length of the detected specimen, $v$ is the ultrasonic propagation velocity in the detected specimen, $t_{1}$ and $t_{2}$ are the propagation time of the first wave and the second wave, respectively.

According to the eqs. (2) and (3), it can be got:

$$
t_{0}-t_{1}=\frac{L}{v_{0}}-\frac{L}{v}
$$

According to the eqs. (3) and (4), the length of the detected sample $L$ can be written:

$$
L=\frac{t_{2}-t_{1}}{2} v
$$

According to the eqs. (2), (5) and (6), the ultrasonic propagation velocity $v$ in the detected specimen can be expressed:

$$
v=\frac{2 t_{0}-3 t_{1}+t_{2}}{t_{2}-t_{1}} \cdot \frac{L_{0}}{t_{0}}
$$

Therefore, as long as the propagation time $t_{0}$ in the cou-

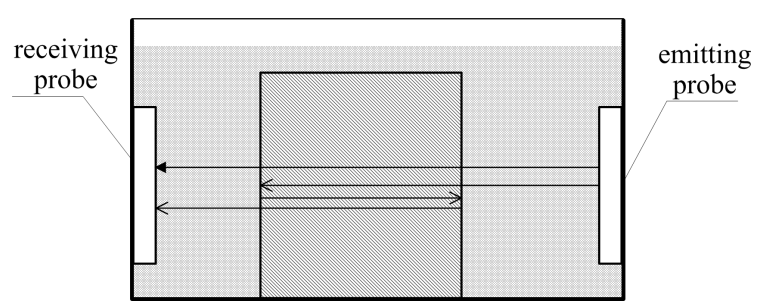

Fig. 1 Principle diagram of testing vermicular graphite percent based on ultrasonic longitudinal wave. pling agent, the propagation time of the first wave and the second wave $t_{1}$ and $t_{2}$, and the distance $L_{0}$ between the emitting probe and the receiving probe were measured, the ultrasonic propagation velocity $v$ in the detected specimen could be calculated according to the eq. (7). Here the distance $L_{0}$ can be kept invariable and be accurately measured in advance. Then, the vermicular graphite percent $\eta$ could be achieved according to certain specific relation established by experiments.

\section{Ultrasonic Wave System for Fast Detecting Vermicu- lar Graphite Percent}

According to the above principle, an ultrasonic wave system was constructed to fast detect the vermicular graphite percent in cast iron. As shown in Fig. 2, the system is mainly composed of the sink, ultrasonic emitting probe, ultrasonic receiving probe, high-frequency signal generator, ultrasonic signal acquisition unit, coupling agent, temperature controller and computer. The emitting and receiving probes were coaxially installed on the opposite sides of the sink, and the distance is $300 \mathrm{~mm}$. FLUKE294 was used to be high-frequency signal generator and can generate arbitrary wave with the frequency of $0.1 \mathrm{~Hz} 40 \mathrm{MHz}$. The sine signal generated by FLUKE294 was applied on the ultrasonic emitting probe, and an ultrasonic longitudinal wave with frequency of $5 \mathrm{MHz}$ will generate due to the piezoelectric effect, and will propagate through the coupling agent and cast iron specimen. Digital oscilloscope DPO4054 with the sampling rate of $2.5 \mathrm{GS} / \mathrm{s}$ was used to be the ultrasonic signal acquisition unit, which can meet the requirement of continuous real-time acquisition of ultrasonic signal. The accuracy of the temperature controller is $\pm 0.5^{\circ} \mathrm{C}$ and the coupling agent is pure water.

The software was written by LabVIEW. On the one hand, it can launch the FLUKE294 and thus generate the sine signal to stimulate the ultrasonic longitudinal wave. On the other hand, it can automatically calculate the propagation time $t_{0}$, $t_{1}, t_{2}$ and the propagation velocity $v$, and then the vermicular graphite percent according to a specific relation.

The working process is as follows. (1) The heating body was launched, and the temperature of the sink was kept at

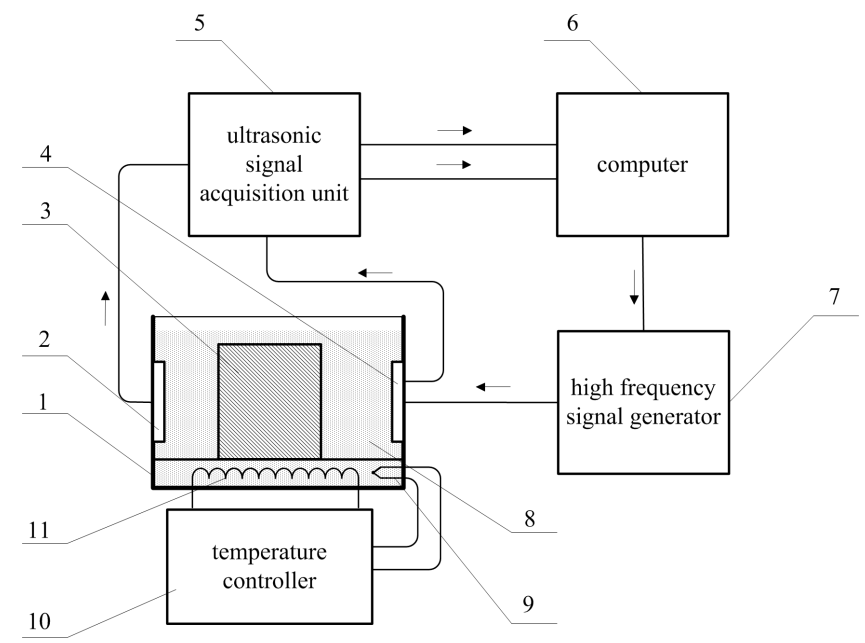

Fig. 2 Ultrasonic wave system for detecting the vermicular graphite percent in cast iron. 
$25^{\circ} \mathrm{C} \pm 0.5^{\circ} \mathrm{C}$ by the temperature sensor and controller. (2) Under the control of computer, FLUKE294 generated a high-frequency sine wave as the driving signal of the ultrasonic emitting probe. (3) On the one hand, the DPO4054 acqured the driving signal and transmitted it to computer, which is considered as the initial reference signal. On the other hand, the ultrasonic longitudinal wave was simulated by the emitting probe, and propagated through the pure water. (4) The ultrasonic longitudinal wave was accepted and transformed into a voltage signal by the receiving probe. The propagation time $t_{0}$ in the pure water will be calculated by computer according to this voltage signal and the initial reference signal. (5) After the detected specimen was put into the sink, the ultrasonic longitudinal wave was simulated again, and the initial reference signal, the first wave signal and the second wave signal were saved by computer, respectively. (6) The propagation time $t_{1}$ and $t_{2}$ were calculated according to these three signals, and then the propagation velocity $v$ in the specimen was calculated according to the eq. (7), and in the end the vermicular graphite percent was given.

\section{Experiment and Analysis}

\subsection{Specific relation between vermicular graphite per- cent and propagation velocity}

Medium frequency induction furnace was used to smelt the cast iron. At $1420^{\circ} \mathrm{C}-1450^{\circ} \mathrm{C}$, the molten cast iron was modified by using different amount of rare earth magnesium titanium alloys, and was poured into the coarse samples at $15 \mathrm{~min}$ utes after modification treatment. The nominal chemical compositions of the molten cast iron were as follows: $w(\mathrm{C})$ : $3.6 \%-3.9 \%, w(\mathrm{Si}): 2.2 \%-2.5 \%, w(\mathrm{Mn}) \leq 0.5 \%, w(\mathrm{P}) \leq$ $0.06 \%$ and $w(\mathrm{~S}) \leq 0.02 \%$.

For one thing, the metallographic specimen was got from the coarse sample, and the vermicular graphite percent was measured by the quantitative metallographic analysis software of Olympus-GX51 microscope. For another, the coarse samples were machined into the detected specimens with the diameter of $40 \mathrm{~mm}$ and $50 \mathrm{~mm}$ and the length of $40-60 \mathrm{~mm}$. The propagation velocity of ultrasonic longitudinal wave in each detected specimen was measured five times, and the mean value was taken as the available result. The comparative measuring results were showed Table 1.

Table 1 Comparative results of vermicular graphite percent and propagation velocity.

\begin{tabular}{|c|c|c|c|c|c|c|c|c|}
\hline \multirow{2}{*}{ No. } & \multirow{2}{*}{$\eta^{*}(\%)$} & \multicolumn{6}{|c|}{$v\left(\mathrm{~m} \cdot \mathrm{s}^{-1}\right)$} & \multirow{2}{*}{ C.V (\%) } \\
\hline & & $v_{1}$ & $v_{2}$ & $v_{3}$ & $v_{4}$ & $v_{5}$ & $\bar{v}$ & \\
\hline 1 & 95.55 & 5287.43 & 5290.39 & 5291.26 & 5288.63 & 5269.83 & 5285.51 & 0.17 \\
\hline 2 & 93.27 & 5301.51 & 5331.71 & 5306.49 & 5304.34 & 5309.92 & 5310.79 & 0.23 \\
\hline 3 & 90.98 & 5345.93 & 5350.06 & 5346.33 & 5312.52 & 5330.76 & 5337.12 & 0.29 \\
\hline 4 & 90.49 & 5333.68 & 5353.04 & 5342.48 & 5338.73 & 5346.46 & 5342.88 & 0.14 \\
\hline 6 & 85.01 & 5412.96 & 5407.72 & 5414.14 & 5406.83 & 5403.31 & 5408.99 & 0.08 \\
\hline 7 & 84.89 & 5414.49 & 5400.56 & 5421.75 & 5417.25 & 5404.86 & 5411.78 & 0.16 \\
\hline 8 & 84.31 & 5426.79 & 5410.01 & 5422.4 & 5422.03 & 5415.23 & 5419.29 & 0.12 \\
\hline 9 & 83.38 & 5432.03 & 5437.05 & 5415.27 & 5437.37 & 5435.71 & 5431.48 & 0.17 \\
\hline 10 & 80.35 & 5490.38 & 5469.61 & 5481.14 & 5462.36 & 5458.69 & 5472.43 & 0.24 \\
\hline 11 & 77.87 & 5528.13 & 5493.02 & 5492.79 & 5520.16 & 5504.87 & 5507.79 & 0.29 \\
\hline 13 & 73.39 & 5586.27 & 5554.69 & 5580.8 & 5569.10 & 5587.51 & 5575.67 & 0.25 \\
\hline 14 & 72.22 & 5605.70 & 5570.75 & 5605.17 & 5585.91 & 5604.01 & 5594.31 & 0.28 \\
\hline 15 & 70.21 & 5627.87 & 5635.16 & 5622.31 & 5622.51 & 5630.09 & 5627.59 & 0.12 \\
\hline 16 & 67.95 & 5672.68 & 5678.24 & 5656.42 & 5644.81 & 5680.35 & 5666.52 & 0.27 \\
\hline 17 & 66.90 & 5692.04 & 5691.85 & 5683.54 & 5666.84 & 5691.13 & 5685.08 & 0.19 \\
\hline 18 & 62.65 & 5722.41 & 5697.39 & 5706.74 & 5694.37 & 5699.8 & 5704.14 & 0.20 \\
\hline 19 & 59.54 & 5709.50 & 5700.57 & 5715.80 & 5722.51 & 5696.19 & 5708.91 & 0.19 \\
\hline 20 & 57.31 & 5695.07 & 5701.71 & 5729.1 & 5716.08 & 5719.69 & 5712.33 & 0.24 \\
\hline 21 & 55.48 & 5717.50 & 5718.82 & 5707.38 & 5730.96 & 5700.95 & 5715.12 & 0.21 \\
\hline 22 & 53.16 & 5717.06 & 5711.57 & 5737.18 & 5712.08 & 5715.47 & 5718.67 & 0.19 \\
\hline 23 & 50.74 & 5729.22 & 5733.25 & 5721.84 & 5714.12 & 5713.43 & 5722.37 & 0.15 \\
\hline 24 & 48.31 & 5717.73 & 5729.05 & 5708.37 & 5733.42 & 5741.88 & 5726.09 & 0.23 \\
\hline 27 & 42.86 & 5723.76 & 5739.52 & 5743.71 & 5736.71 & 5728.43 & 5734.43 & 0.14 \\
\hline 28 & 40.70 & 5734.88 & 5742.84 & 5732.9 & 5732.08 & 5745.95 & 5737.73 & 0.11 \\
\hline 29 & 35.79 & 5757.22 & 5726.43 & 5734.45 & 5745.9 & 5762.22 & 5745.24 & 0.26 \\
\hline 30 & 34.45 & 5760.91 & 5755.96 & 5756.15 & 5730.57 & 5732.85 & 5747.29 & 0.25 \\
\hline
\end{tabular}

Note: $\eta^{*}$ was got by quantitative metallographic analysis software of Olympus-GX51 microscope. 


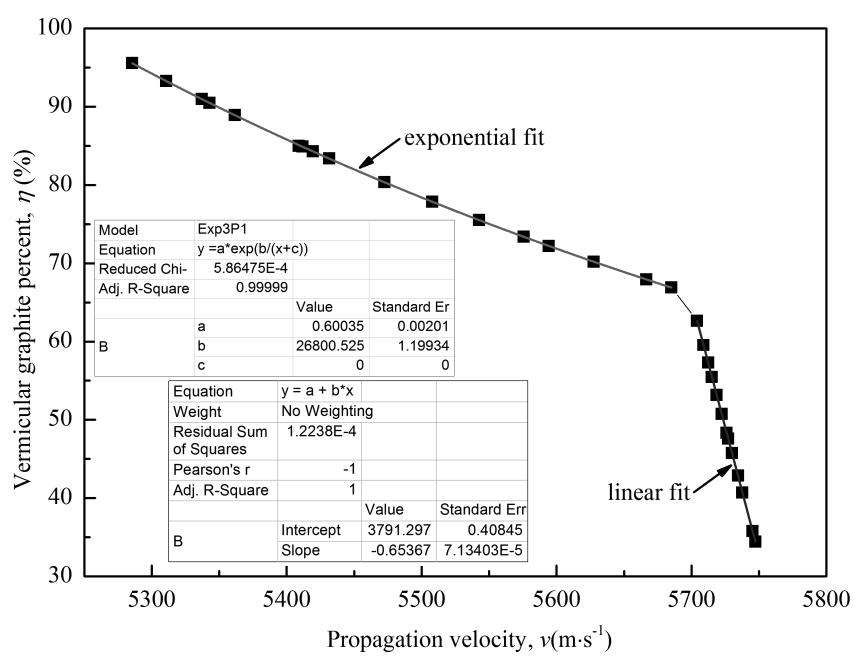

Fig. 3 Relation between vermicular graphite rate and propagation velocity.

The coefficient of variation in mathematical statistics was introduced to evaluate the repeatability of the ultrasonic wave testing system, and it was calculated according to the eq. (8). ${ }^{13)}$

$$
\left\{\begin{array}{l}
\mathrm{C} . \mathrm{V}=\frac{\sigma}{\bar{x}} \times 100 \% \\
\sigma=\sqrt{\frac{\sum_{i=1}^{n}\left(x_{i}-\bar{x}\right)^{2}}{n}}
\end{array}\right.
$$

where, $\mathrm{C} . \mathrm{V}$ is the coefficient of variation, $\sigma$ is the standard deviation, $\bar{x}$ is mean value, $\bar{x}=\sum_{i=1}^{n} x_{i} / n, x_{i}$ is the measuring result at the time $i$, and $n$ is the times of the measurement.

The calculated results according to equation (8) were also shown in Table 1. It can be seen the coefficient of variation is below $0.3 \%$, which indicates the repeatability of the system is very good.

Figure 3 showed the relation between the vermicular graphite percent of cast iron and the propagation velocity of ultrasonic longitudinal wave.

As shown in Fig. 3, when the vermicular graphite rate is below $66 \%$, there is a linear relationship between them. Once the vermicular graphite percent is more than $66 \%$, it is an approximate exponential relationship. Therefore, a specific relation between them can be got by the curve fitting, namely:

$$
\begin{cases}\eta=-\frac{v}{1.53}+\frac{5800}{1.53} & v \in(5700,5800] \\ \eta=0.6 \cdot \exp \left(\frac{26800}{v}\right) & v \in[5250,5700]\end{cases}
$$

where, $\eta$ is the vermicular graphite percent in cast iron, $\%, v$ is the ultrasonic longitudinal propagation velocity in cast iron, $\mathrm{m} \cdot \mathrm{s}^{-1}$.

So, as long as the propagation velocity $v$ was measured by this system, the vermiculation graphite percent $\eta$ could be calculated according to the eq. (9).
Table 2 Comparative results of vermicular graphite percent measured by

\begin{tabular}{|c|c|c|c|c|c|c|c|}
\hline \multirow{2}{*}{ No. } & \multicolumn{3}{|c|}{ propagation time $(\mu \mathrm{s})$} & \multirow{2}{*}{$v\left(\mathrm{~m} \cdot \mathrm{s}^{-1}\right)$} & \multicolumn{2}{|r|}{$\eta$} & \multirow{2}{*}{ error $(\%$} \\
\hline & $t_{0}$ & $t_{1}$ & $t_{2}$ & & system & metallographic & \\
\hline 1 & 133.57 & 112.51 & 129.16 & 5285.21 & 95.58 & 96.39 & 0.84 \\
\hline 2 & 133.27 & 106.96 & 127.65 & 5317.41 & 92.69 & 93.84 & 1.23 \\
\hline 3 & 133.75 & 104.72 & 126.91 & 5407.84 & 85.19 & 85.89 & 0.81 \\
\hline 4 & 133.37 & 104.42 & 126.55 & 5423.05 & 84.02 & 81.25 & 3.41 \\
\hline 5 & 133.57 & 111.61 & 127.79 & 5561.82 & 74.27 & 72.89 & 1.89 \\
\hline 6 & 133.73 & 112.14 & 127.79 & 5621.93 & 70.54 & 70.15 & 0.56 \\
\hline 7 & 133.49 & 109.91 & 126.81 & 5679.13 & 67.24 & 69.43 & 3.16 \\
\hline 8 & 133.32 & 103.84 & 124.88 & 5703.99 & 62.75 & 62.29 & 0.74 \\
\hline 9 & 133.53 & 103.98 & 125.01 & 5706.99 & 60.79 & 60.20 & 0.98 \\
\hline 10 & 133.52 & 106.43 & 125.67 & 5716.01 & 54.90 & 55.92 & 1.83 \\
\hline
\end{tabular}
the system and quantitative metallographic method.
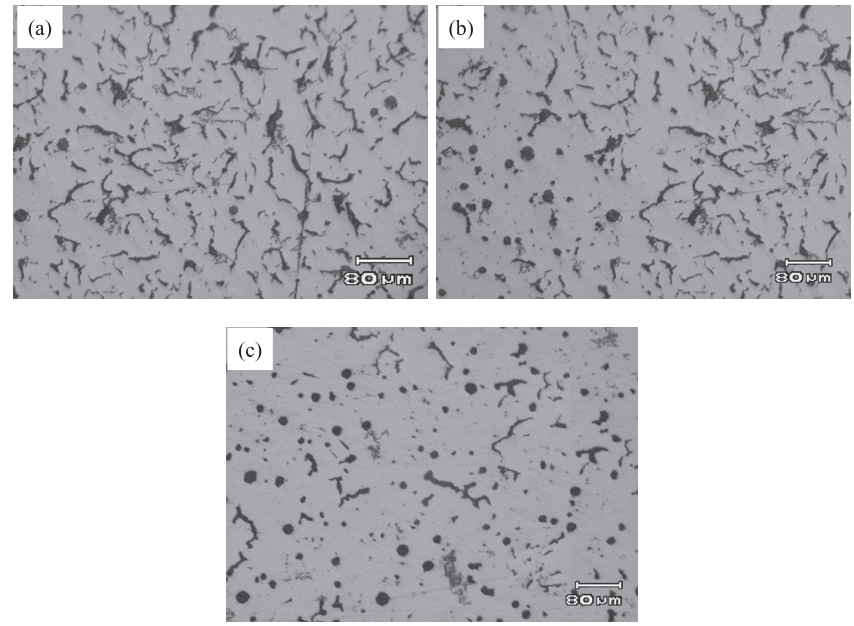

Fig. 4 Vermicular graphite percent and propagation velocity of typical detected specimen: (a) $\eta=90 \%, v=5717 \mathrm{~m} \cdot \mathrm{s}^{-1}$, (b) $\eta=85 \%, v=5592 \mathrm{~m} \cdot \mathrm{s}^{-1}$, (c) $\eta=70 \%, v=5530 \mathrm{~m} \cdot \mathrm{s}^{-1}$.

\subsection{Experimental verification}

The specimens with different vermicular graphite percent were used to verify the accuracy of the system. The vermicular graphite percent was measured by the system and quantitative metallographic method, respectively, and the results were shown in Table 2. Figure 4 is the comparison between the vermicular graphite percent and the ultrasonic longitudinal propagation velocity of typical detected specimens.

Table 2 indicated the error is below $4 \%$, that is to say, the accuracy of detecting the vermicular graphite percent by the system is above $96 \%$.

The error can be attributed to two aspects. One is due to the difference between the regional detection of quantitative metallographic and the global measurement of the ultrasonic system. Another is owing to the accuracy of calculating the propagation time and the model precision of calculating the vermicular graphite percent according to the eq. (9).

When the model of calculating the vermicular graphite percent was found out, the number of experimental specimens was very few (only 30 specimens), and thus the error of the fitting eq. (9) will be a little large. But the error will gradually decrease with the increase of the number of the specimens. In 
addition, besides the vermicular graphite percent, the ultrasonic propagation velocity in the specimen was also affected by the graphite shape, matrix microstructure, impurities and defects, which will lead to the error. More importantly, the propagation velocity was calculated by the eq. (7) according to three propagation time $t_{0}, t_{1}$ and $t_{2}$ where the order of magnitude is microsecond. So, a small fluctuation of the propagation time will cause a large change of the propagation velocity, and thus affect the calculation result of the vermicular graphite percent.

\section{Conclusions}

(1) An ultrasonic longitudinal wave system was constructed to fast detect the vermicular graphite percent in cast iron. It is mainly composed of the sink, ultrasonic emitting probe, ultrasonic receiving probe, high-frequency signal generator (FLUKE294), ultrasonic signal acquisition unit (DPO4054), coupling agent, temperature controller and computer.

(2) A specific relation was got by experiments. When the propagation velocity is more than $5700 \mathrm{~m} \cdot \mathrm{s}^{-1}$, it has a linear relationship between the vermicular graphite percent $\eta$ and the propagation velocity $v$, namely $\eta=-v / 1.53+5800 / 1.53$. When the velocity is less than $5700 \mathrm{~m} \cdot \mathrm{s}^{-1}$, it is approximately exponential, namely $\eta=0.6 \cdot \exp (26800 / v)$.

(3) The coefficient of variation for the propagation velocity in the system is below $0.3 \%$, which indicates the repeatability of the system is very good. The error of comparative experi- ments between the system and the quantitative metallographic method is below $4 \%$, which proves the accuracy of the system is more than $96 \%$.

\section{Acknowledgements}

This work was supported by Foundation for University Key Teacher of Heilongjiang Province of China (12541107). The authors would like to acknowledge it.

\section{REFERENCES}

1) C. H. Lim and B. C. Goo: Met. Mater. Int. 17 (2011) 199-205.

2) T. Sjögren, P. Vomacka, and I. L. Svensson: Int. J. Cast Metal. Res. 17 (2004) 65-71.

3) M. C. Firican and I. Riposan: Adv. Mater. Res. 1128 (2015) 72-79.

4) J. Erneman, M. Schwind, L. Nylöf MSc, J. Nilsson, H. Andrén and J. Agren: Metall. Mater. Trans. A 36 (2005) 2595-2600.

5) P. I. Popov and I. G. Sizov: Met. Sci. Heat Treat. 48 (2006) 272-275.

6) D. N. Collins and W. Alcheikh: J. Mater. Process. Tech. 55 (1995) 8590.

7) X. J. Sun, Y. X. Li and X. Chen: J. Mater. Process. Tech. 200 (2008) 471-480.

8) V. K. Kachanov, I. V. Sokolov, R. V. Kontsov, and L. V. Voronkova: Meas. Tech. 54 (2012) 1280-1284.

9) T. Uchimoto, T. Takagi and T. Abe: Mater. Trans. 51 (2010) 1114-1119.

10) Z. G. Wang and L. W. Meyer: Exp. Mech. 50 (2010) 1061-1074.

11) T. Sjögren and I. L. Svensson: Int. J. Cast Metal. Res. 17 (2004) 271279.

12) W. Gaudig, R. Mellert, U. Weber and S. Schmauder: Comp. Mater. Sci. 28 (2003) 654-662.

13) X. He and S. O. Oyadiji: J. Mater. Process. Tech. 119 (2001) 374-378. 